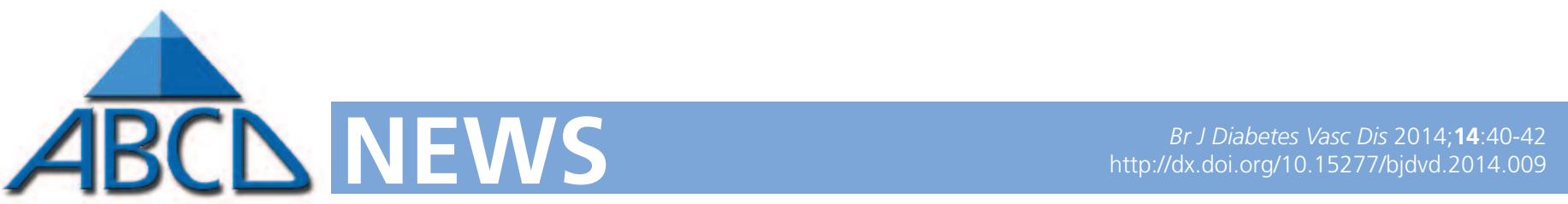

\title{
ABCD now on N3: The ABCD nationwide exenatide QW audit
}

\author{
Dr Robert EJ Ryder \\ Consultant Diabetologist, Clinical Lead, ABCD nationwide audits, City Hospital, Birmingham, UK
}

March 2014 sees, in the same month, two significant milestones for the Association of British Clinical Diabetologists (ABCD). The first is the launch of its own journal as it takes over the British Journal of Diabetes \& Vascular Disease in the current issue. ${ }^{1}$ The second is the establishment of ABCD's presence on N3 with the launch of the ABCD nationwide exenatide QW audit.

\section{What is N3 and why a presence on it for $A B C D$ ?}

N3 is the national broadband network for the National Health Service (NHS), connecting all NHS locations, in particular linking acute hospitals and GP surgeries. The important thing from ABCD's point of view is that it is the official secure place for storing NHS patient data and therefore the most appropriate and secure place for holding our nationwide audit data in the future.

\section{Previous $A B C D$ nationwide audits}

$A B C D$ has undertaken a number of nationwide audits since $2004 .^{2}$ However it was really the $A B C D$ nationwide exenatide audit, ${ }^{2}$ followed by the still on-going nationwide liraglutide audit, ${ }^{2}$ which established the pattern for the future. The nationwide exenatide audit collected data on 6,717 patients from 315 contributors and 126 centres. As of January 2014 the liraglutide audit had 6,213 patients from 117 centres and 550 contributors. From these audits we learned much. For example, that patients in the UK treated with exenatide and liraglutide were heavier and more poorly controlled than patients in the clinical trials of these agents. ${ }^{3}$ That the agents were often used off licence and outside of NICE guidelines, in particular with insulin; we were able to establish that in this situation they could be safe and effective. ${ }^{4,5}$ We demonstrated that it was safer to add the glucagon-like peptide-1 (GLP-1) receptor agonist to insulin and then tail off the insulin, rather than to discontinue the insulin at the commencement of exenatide or liraglutide in order to stay on licence or within guidelines, which could often be an unsafe thing to do. ${ }^{5}$ We were able to demonstrate that patients with professional driving licences achieved some success at avoiding insulin through the use of GLP-1 receptor agonists. ${ }^{6}$ We have been able to collate audit data on liraglutide use outside licence in mild renal impairment and report that efficacy has been preserved and no safety concerns reported. ${ }^{7}$ And most recently, we were able to contribute to the debate over whether GLP-1 related therapies might be associated with pancreatic damage - we found no such signal in our nationwide audits. ${ }^{8,9}$

\section{Previous audit tools}

For the nationwide exenatide audit an online tool built in-house was utilised. But because of insufficient security, users had to enter anonymised data with codes for each patient. The problem with that was that it required the user to keep a record of which codes represented which patient, which was very much less than ideal. For the liraglutide audit a professional tool was built, initially in the form of a sophisticated downloadable Excel spread sheet, but this was migrated in 2011 to an on-line tool in a secure area. Whilst this was sufficiently secure, using the same technology as used by banks, it was nevertheless less than the ideal place, the latter being N3. Thus ABCD embarked on a project to establish an online tool on N3 and this month sees the launch of that tool and ABCD's presence on N3 for all such activities in the future.

\section{Exenatide QW audit}

The web-based tool on N3 ${ }^{10}$ is very easy to use. Centres may register to join the audit and designated contributors from each cen- tre are each given individualised logons that give access to the on-line tool for their centre. The tool facilitates the collection of routine clinical information of patients at each of their visits. Centres may download their own data for local analysis and audit. However, their data are available at the same time in an anonymised form for the national audit. A sophisticated analysis tool has been built, such that the data can be grouped at intervals specified by whoever is analysing the data - for instance 2 monthly, 3 monthly, 4 monthly, 6 monthly, etc. The tool is so easy to use that data can be entered into the tool live in clinic, or alternatively it can collected onto paper proformas for entry into the tool later. The proformas can be printed from a downloadable PDF file, or there is a facility for ordering paper forms to be supplied by post. Whilst the audit is particularly for ABCD members, it is open to non-members and, indeed, anybody or centre using exenatide QW may join.

\section{Future audits}

Future audits already agreed or under consideration include:

Nationwide dapagliflozin audit: This audit is particularly important with dapagliflozin being the first of a new class of drugs for diabetes, the Sodium/GLucose coTransporter 2 (SGLT2) inhibitors. As with the ABCD GLP-1 receptor agonist audits, it may be that patients being treated with the new class in real clinical practice will be different from those in clinical trials and outcomes may also be different both in terms of safety and efficacy. The audit is definitely going ahead and the questionnaire for the on-line tool is currently being developed. It will be hosted by ABCD on N3, as with the exenatide QW audit, and those taking part in the exenatide QW audit should find the process of the dapagliflozin audit very similar because of the similarity of the on-line 
tools. It is hoped that the dapagliflozin audit will launch as soon as the exenatide QW audit is well underway. It is also hoped that in due course the dapagliflozin audit will be enhanced by the Primary Care Diabetes Society joining in.

Nationwide degludec audit: This audit is in an advanced state of consideration. With many older established insulins in common usage, it will be important to try to gain insight into degludec in real clinical practice by attempting to record the routine data on all patients treated with this new insulin, if possible, so that the most accurate picture of it can be obtained. By pooling the data nationally we will hopefully learn more quickly from the shared experience. In clinical trials degludec was associated with less hypoglycaemia than other insulins and allowed for flexible dosing. The audit may give insight into whether these potential advantages translate in real clinical practice. The tool will be hosted on a tool very similar to that used in the liraglutide audit, so those taking part in that audit will find it particularly easy.

Possible nationwide lixisenatide audit: $A B C D$ is giving consideration to such an audit given the fact that lixisenatide does seem to be different from some of the other GLP-1 receptor agonists, and it will be of interest and clinically useful to see how these differences translate in real clinical practice.

\section{Does your centre use exenatide QW?}

If yes, then please register for the nationwide audit, as described above, through http://www.diabetologists-abcd.org.uk/n3/ ExenatideQW_Audit.htm. Please remember, if contributing, you will be able to analyse your local data easily, including use of the sophisticated tool for grouping the data. Also, all contributors will be listed in publications and presentations arising from data submission.

\section{References}

1. Walton C. Entering a new era. Br J Diabetes Vasc Dis 2014;14(1):1

2. The history of the $A B C D$ nationwide audit programme. Available at http://www.diabetologists-abcd.org.uk/n3/previous_ABCD_ audits.htm (2014, accessed 3 March 2014)

3. Ryder B, Thong $K$, the ABCD nationwide exenatide and nationwide liraglutide audit contributors. Findings from the Association of British Clinical Diabetologists ( $A B C D$ ) nationwide exenatide and liraglutide audits. In Hot topics in diabetes, Vora J, ed. Synergy, London, 2012. Available at http://www. diabetologists-abcd.org.uk/GLP1_Audits/ABCD_Hot_ Topics_2012.pdf (2012, accessed 3 March 2014)

4. Thong KY, Jose B, Sukumar $\mathrm{N}$ et al. Safety, efficacy and tolerability of exenatide in combination with insulin in the Association of British Clinical Diabetologists nationwide exenatide audit. Diab Obes Metab 2011;13: 703-10. http://dx.doi.org/10.1111/.1463-1326.2011.01393.x

5. Thong KY, Jose B, Blann AD et al. Response at 3 months to insulin dose decisions made at exenatide initiation in the Association of British Clinical Diabetologists (ABCD) nationwide exenatide audit. Diab Res Clin Pract 2011:93:e87-91 http://dx.doi.org/10.1016/..diabres.2011.05.015

6. Thong KY, Cull ML, Ryder REJ et al. Insulin avoidance and treatment outcomes among patients with a professional driving licence starting glucagon-like peptide 1 (GLP-1) agonists in the Association of British Clinical Diabetologists $(A B C D)$ nationwide exenatide and liraglutide audits. Diabet Med 2012; 29(5):690-92.

http://dx.doi.org/10.1111/j.1464-5491.2011.03475.x

7. Thong KY, Walton $C$, Ryder REJ, the ABCD nationwide liraglutide audit contributors. Safety and efficacy of liraglutide $1.2 \mathrm{mg}$ in patients with mild and moderate renal impairment:the $A B C D$ nationwide liraglutide audit. Practical Diabetes 2013:30(2):71-6. http://dx.doi.org/10.1002/ pdi.1748

8. Ryder REJ, Thong KY, Blann AD et al. Liraglutide pancreatitis: The $A B C D$ nationwide liraglutide audit. $\mathrm{Br} /$ Diabetes Vasc Dis 2013;13:253-9.

http://dx.doi.org/10.1177/1474651413502685

9. Ryder REJ, Sen Gupta P, Thong KY et al. The Association of British Clinical Diabetologists nationwide exenatide and liraglutide audits suggest a low incidence of acute pancreatitis. Response to Robson. Incretins and pancreatitis - what happens next? A personal viewpoint. Diabet Med 2013;30(12):1510-11 http://dx.doi.org/10.1111/dme.12336

10. $A B C D$ nationwide exenatide $Q W$ audit on N3. Available at http://www.diabetologistsabcd.org.uk/n3/ExenatideQW_Audit.htm (2014, accessed 3 March 2014)

Address for correspondence:

Dr Bob Ryder, Diabetes and Endocrine Unit, City Hospital, Dudley Road, Birmingham B18 7QH, UK

E-mail: bob.ryder@nhs.net

Conflict of interest: REJR has received speaker fees, consultancy fees and/or educational sponsorship from a number of companies including in alphabetical order, Bristol Myers Squibb / Astra Zeneca Alliance, Eli Lilly, GlaxoSmithKline, Novo Nordisk, Sanofi-Aventis and Takeda.

\title{
Metamorphosis: from the Newsletter to the Journal
}

\author{
Dr Peter Winocour \\ Consultant Diabetologist and ABCD executive, Queen Elizabeth II Hospital, Welwyn Garden City, Herts, UK
}

Timelines can often offer a helpful perspective on the workings of the NHS

The Association of British Clinical Diabetologists (ABCD) was founded in 1997, at a time of yet further restructuring of the NHS. Our first Newsletter appeared in the autumn of 2002 just after the launch of the National Service Framework (NSF) for Diabetes, which set out ambitious 10 year standards with a later delivery document and strategy. Our first editor Peter Daggett served for over 5 years and offered challenges to us and the broader systems we worked in, using his 'Controversy' section to deliver exactly what 'it said on the tin'. More specialists was the mantra - we need to keep on banging the drum whilst recognising that the role of the diabetologist is expanding to serving the population through service integration and the support of primary care in addition to the key role in specialist areas of diabetes, endocrinology and the ongoing commitment to acute and general internal medicine.

The difference of views as to whether we will develop as a specialty by embracing the 'Future Hospital' and 'Shape of Training' themes of generalism or are better served by focusing on work as specialists will become clearer with time. I nail my colours to the mast by promoting the concept of a pool of consultants in diabetes and endocrinology expanding posts in all hospital settings, enabling the contribution to acute general internal medicine to be paralleled 
by specialist service expansion. Whether prescient or through chance, I led a discussion at the Spring 2002 meeting and of fered conclusions to enable an $A B C D$ response to the NSF standards which Peter Daggett published in the Newsletter.

We were sceptical of the standard to reduce the risk of type 2 diabetes without societal change. The only change since has been in the wrong direction in terms of incident obesity and a $10 \%$ chance of developing type 2 diabetes in adult life. Unselected population screening for diabetes was not supported. The more recent adoption of the NHS cardiovascular disease (CVD) risk check for all aged 45 or more is indeed probably not cost effective if not targeted but is going ahead - let's see how much avoidable morbidity results. We endorsed shared care and have since led the drive for integrated diabetes services - now hopefully the only show in town. We supported an end to cattle market diabetes clinics and strongly pushed for better care for children and young people with diabetes, pushing for mandatory training in diabetes amongst paediatric registrars - sadly never realised. In-patient diabetes was high on the NSF agenda and education of health care professionals about diabetes through an in-patient diabetes specialist nurse was promoted by $A B C D$. The ongoing push spearheaded by Gerry Rayman may prove to be one of the major deliverables for the NSF along with integrated ante-natal diabetes care.

\section{Where are we now?}

So here we are over 10 years on after the NSF delivery document. I think we have spent the decade revising systems and structures and focusing on care processes and measuring them. We have more data on diabetes from the National Diabetes Audit than we know what to do with. The local information may or may not be utilised depending on the drive and support of commissioners and public health - not always guaranteed to be present in large quantity.

I have always felt that, whilst our expertise in supporting the care of patients with type 1 diabetes required separation and commissioning, the current standard approach to clinic based services would not deliver the best results. Similarly, the understandable suggestion that type 2 diabetes required predominant primary care recognised firstly the high prevalence and the clear fact that many patients could be adequately managed through primary care support and education using best practice guidelines. However, by presenting type 2 diabetes as a condition managed by algorithm, I think we have done many patients a disservice. The evidence base for the guidance is not there; the individualization of care is absolutely necessary but not at all straightforward. When we consider the option for the 12 classes of agent in managing type 2 diabetes and delegate the task to many excellent practice nurses, I can understand the failure to shift the adverse outcomes shown in the National Diabetes Audit and the recent description of therapeutic inertia in the primary care setting. The lessons of the need to front end intensify treatment of diabetes and related CVD risk in newly diagnosed type 2 diabetes especially when younger, whilst taking a more measured conservative approach in older patients with established CVD, is I think yet to factor into routine primary care of type 2 diabetes. If specialists rely on being 'complicationologists' for type 2 diabetes whilst the system does not look to do more earlier, I anticipate more complicated diabetes in the next decade. There is a way forward and it is NOT through the separation of care into different sectors. Diabetologists embracing new technologies and smarter means of behaviour change may make the difference. Since Roy Taylor disproved the dogma that type 2 diabetes is inevitably linked with progressive beta cell damage, the opportunity exists to reverse the tide of diabetes and complications BUT it still needs more front end care.

John Wales was the first chairman of $A B C D$. In his valedictory in our first Newsletter talked of the need to work closely with the Colleges of Physicians and in the intervening period we have been represented on the College Council and taken a lead role in delivery of the SCE examination, and serving as rotating secretary - chairman on the Joint Specialty Certificate for Diabetes and Endocrinology.

Whilst the association has gone from strength to strength over the 12 years, the Newsletter also evolved. Mark Savage took on the mantle of editor in 2008. Like Peter Daggett, Mark was not backward in coming forward in expressing his views, and when I took over from Mark when he escaped to $\mathrm{Oz}, \mathrm{I}$ also relished the opportunity to 'offer a view'.
I think the time is ripe for a move from the more conversational tone of the Newsletter to the more disciplined requirements of our new journal, which I am certain will prove a great success and will be a testament to the vision of our outgoing chairman Chris Walton. However in establishing the values and objectives of the main substance of the journal, we have agreed that the ethos of the Newsletter should remain, with the $A B C D$ News section taking a topical view of the challenges we face in balancing our commitments to providing best quality diabetes care with the other aspects of our day jobs.

\section{The future role of $A B C D$}

I think $A B C D$ has grown up. As the UK specialist diabetes association, we could look to our role on the wider stage. The JBDS guidelines have resonance internationally and we need to promote this important output beyond our shores. We are now supporting an expansion into subspecialist areas through our collaboration with the Renal Association.

We can and should take an active role in supporting better UK hospital care. Many years ago Chris Walton and others had a dream that peer review of diabetes services was a possibility. Given the multidisciplinary nature of the team this was always going to be a challenge. However through peer review of diabetes services for children and young people, and the likely expansion to transitional care of adolescents, there is the first opportunity to start the process. The recent engagement between $A B C D$, Diabetes UK and the Care Quality Commission to support in-patient hospital diabetes peer review initially is an exciting opportunity for the years ahead.

One advertising slogan stated 'the future is bright the future is orange'. This is only partly correct - the future is indeed bright but the future is actually a pyramid of multiple hues of blue!

Address for correspondence: Dr Peter Winocour, Queen Elizabeth II Hospital, Howlands, Welwyn Garden City, Herts, AL7 4HQ, UK E-mail:peter.winocour@nhs.net

Conflict of interest: None 